\section{Vigilancia nutricional en escolares de la ciudad de Santa Fe: educación experiencial con estudiantes de Licenciatura en Nutrición}

\section{Gimena Dezar}

gdezar@unl.edu.ar

\section{Ana Luz Kruger}

krugluzana@hotmail.com

Docentes de la Facultad de Bioquímica

y Ciencias Biológicas. Universidad

Nacional del Litoral, Argentina.
Integración de la docencia y la extensión /

Intervenciones

RECEPCIÓN: 21/06/16

ACEPTACIÓN FINAL: 04/10/16

\section{Resumen}

El presente trabajo relata la experiencia de una propuesta de práctica de extensión de educación experiencial desarrollada por alumnas de $3^{\circ}$ año de la carrera de Licenciatura en Nutrición de la Universidad Nacional del Litoral (UNL). Además, participaron el equipo docente de la cátedra de Epidemiología y Salud Pública, tutores, docentes y directivos de nueve escuelas primarias de la ciudad de Santa Fe, niños y niñas de esas instituciones, sus padres y familias. Desde el equipo docente se viene trabajando en extensión, integrándola con la docencia y la investigación, haciendo hincapié en problemas nutricionales que ocurren en la niñez. En esta dirección, y en concordancia con lo planteado desde la UNL, se intenta contribuir de manera significativa a una mejor calidad y pertinencia universitaria, participando en los procesos de enseñanza y aprendizaje, en la generación de nuevos conocimientos y en la apropiación social de los mismos.

Palabras-clave

- Educación experiencial

- Escolares

- Vigilancia nutricional

\section{Resumo}

Este artigo descreve a experiência de uma proposta de prática de extensão experiencial, desenvolvida por estudantes do terceiro ano do curso de Bacharelado em Nutrição da Universidade Nacional del Litoral (UNL). Participaram também a equipe docente da disciplina de Epidemiologia e Saúde Pública, tutores, professores e diretores de nove escolas primárias da cidade de Santa Fe, crianças dessas instituições, seus pais e famílias. A equipe docente vem trabalhando em extensão, integrando-a à docência e à pesquisa, focando-se nos problemas nutricionais que ocorrem na infância. Neste sentido, e de acordo com as declarações da UNL, pretende-se contribuir significativamente para uma melhor qualidade e maior pertinência universitária, participando nos processos de ensino e aprendizagem, na geração de novos conhecimentos e apropriação social deles.

Palavras-chave

- Educação experiencial

- Escolares

- Vigilância nutricional
Para citación de este artículo

Dezar, G. y Kruger, A. L. (2016). Vigilancia nutricional en escolares de la ciudad de Santa Fe: educación experiencial con estudiantes de Licenciatura en Nutrición. En Revista +E versión digital, (6), pp. 264-271. Santa Fe, Argentina: Ediciones UNL. 


\section{Introducción}

Desde la cátedra de Epidemiología y Salud Pública de la Facultad de Bioquímica y Ciencias Biológicas (FBCB-UNL) se desarrollan desde el año 2010 actividades para la evaluación del estado nutricional de niños en edad escolar de escuelas seleccionadas de la ciudad de Santa Fe. Estas actividades involucran la evaluación mediante antropometría del peso y talla (para la construcción de índices recomendados para la evaluación del estado nutricional) y la realización de encuestas nutricionales. La experiencia, realizada en tres oportunidades, ha brindado resultados de suma importancia y utilidad para que, una vez presentados y puestos a disposición de autoridades sanitarias y educativas, sean utilizados como base para el diseño de políticas públicas sanitarias que contribuyan a mejorar la salud de los niños en nuestra región.

Previamente, durante 2008 y 2009, se trabajó en la evaluación nutricional de todos los niños menores de 5 años del barrio 29 de abril de la ciudad de Santa Fe. Adicionalmente, de manera conjunta con el personal del Centro de Salud Callejón Roca, se hizo un censo de todas las familias que vivían en el barrio.

\subsection{Sistemas de vigilancia en salud}

Un sistema de vigilancia en salud es la producción sistemática de información sobre el comportamiento del continuo saludenfermedad y los factores que lo condicionan, para orientar la toma de decisiones dirigidas a mejorar la calidad de salud de la población. Los sistemas de vigilancia recopilan datos en forma oportuna, sistemática y ordenada, seguidos de un análisis e interpretación para observar o registrar cambios en la tendencia o distribución de los problemas de salud y sus factores asociados. Es decir, se produce información para el diseño de medidas de prevención y control de enfermedades o promoción de salud para una población (Ministerio de Salud de la Nación, 2013).

\subsection{Sistemas de vigilancia nutricional}

Un sistema de vigilancia alimentaria y nutricional es un proceso sistemático de recolección, análisis, interpretación y difusión de datos, usando métodos que se distinguen por ser prácticos, uniformes y rápidos, más que por su exactitud o totalidad, que sirven para observar las tendencias en tiempo, lugar y persona, con lo que pueden observarse o anticiparse cambios para realizar las acciones oportunas, incluyendo la investigación y/o la aplicación de las medidas de control de los problemas alimentarios y nutricionales (Last, 2001).

La importancia de la vigilancia nutricional se fundamenta en la generación de información que permita la toma de decisiones de forma oportuna por un lado, y por otro, que estos se traduzcan en políticas de salud en vistas de mejorar o mantener la salud de la población objetivo en el largo plazo.

\section{El problema a trabajar: malnutrición}

La malnutrición, tanto por déficit como por exceso de peso, es un problema que afecta a gran cantidad de población de todas las edades, que debe ser considerado en si mismo un problema de salud pero que además, está documentado como uno de los determinantes más importantes en la generación, mantenimiento y agravamiento de muchos otros trastornos de salud en la población. En particular en los niños la cantidad de afectados por trastornos nutricionales es de importancia, ya que según los datos de la última encuesta nacional sobre nutrición que se ha realizado (Encuesta Nacional de Nutrición y Salud, 2004) por parte del Ministerio de Salud de la Nación, el 3,8 \% de los menores de 5 años estaba desnutrido, ya sea en forma aguda o crónica, y de éstos, el 4,2 \% tenía "acortamiento", que es indicativo de desnutrición crónica. Adicionalmente, y siempre según la misma fuente, a nivel nacional el 6,6 \% de los niños de la misma edad padecía de obesidad, lo cual es una cifra de importancia, más aún si se tiene en cuenta que este valor se incremente notablemente con la edad.

A su vez, como elemento de la importancia del problema, es conveniente destacar que, más allá del peso específico que tiene el problema de los trastornos nutricionales por sí mismo, cualquier condición adversa en lo nutricional es un factor predisponente y de riesgo para una gran cantidad de problemas de salud asociados sobre todo con la infancia y para retardar y deteriorar el desarrollo de los niños y también debilitar el rendimiento escolar que tendrán. El problema que se pretende abordar es la malnutrición. A partir de los resultados obtenidos con esta experiencia se identificaron los problemas nutricionales de la población de escolares que asisten a las escuelas seleccionadas de la ciudad de Santa Fe. Se confeccionaron informes escritos y se propusieron estrategias para mejorar la alimentación de las familias en general mediante talleres teóricoprácticos de acuerdo con las edades y disponibilidad de tiempo y espacio en las escuelas donde se desarrollaron los mismos.

\section{La experiencia recorrida con los estudiantes}

La malnutrición infantil es un estado donde existe una insuficiencia o exceso de nutrientes que conlleva a diversas patologías. La edad escolar es un período crítico donde deben realizarse exámenes periódicos del estado nutricional. En este estudio se ponderó la prevalencia de malnutrición en escolares de $1^{\circ}$ y $4^{\circ}$ grado en 9 escuelas de la ciudad de Santa Fe, de las cuales 3 cuentan con comedor. Se tomaron medidas antropométricas de peso y talla. Se utilizó el indicador Índice de Masa Corporal para la Edad (IMC/E) para determinar el estado nutricional. Se halló una alta prevalencia de malnutrición por exceso: $30,11 \%$ de sobrepeso y $23,43 \%$ de obesidad en un total de 734 niños evaluados. El bajo peso sólo constituyó un $0,41 \%$ de la muestra. Hubo mayor 


\section{6}

la propuesta tuvo como propósito

fortalecer acciones interinstitucionales

orientadas a contribuir en la

incorporación de hábitos saludables

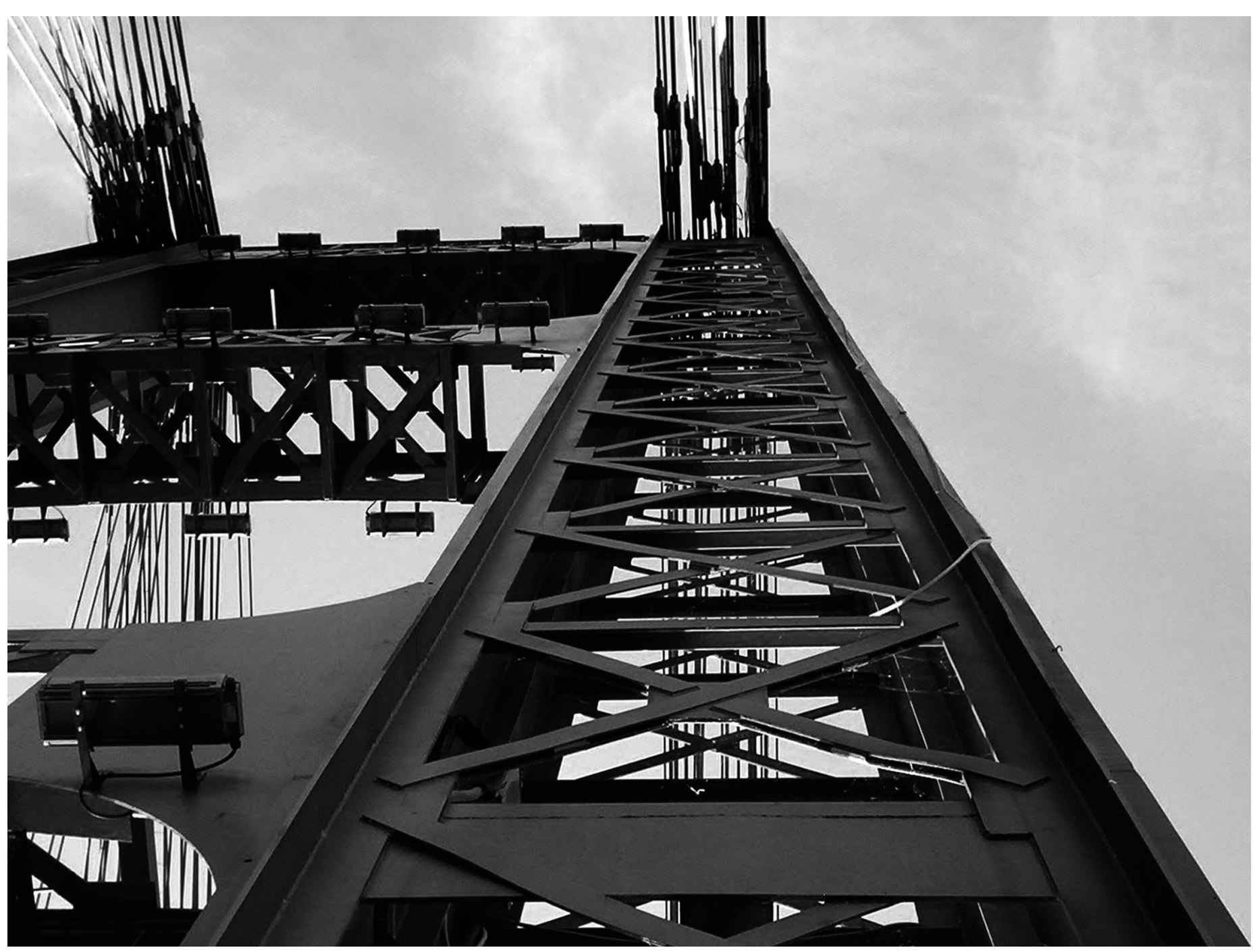

(c) Oscar Dechiara 
proporción de malnutrición por exceso entre los varones y los niños de $4^{\circ}$ grado. Las cifras obtenidas constituyen un punto de partida para la realización de intervenciones que busquen prevenir y mitigar esta situación.

A partir de este diagnóstico realizado en las escuelas, los estudiantes del $3^{\circ}$ año de Licenciatura en Nutrición pudieron hacer los informes individuales de los niños y niñas para ser entregados a los padres de cada uno de ellos a través de la escuela, con el resultado de la evaluación nutricional por antropometría. Además elaboraron y presentaron informes generales a las autoridades de la Escuela para conocer la situación nutricional general y la evolución que esta situación tiene cada año, lo que permitió fortalecer las actividades tendientes a promover una buena condición nutricional de los escolares. Por último, confeccionaron folletos con información sobre Educación Alimentaria Nutricional que fueron entregados a los niños del establecimiento sobre conocimientos de nutrición que puedan servir para cambiar hábitos poco saludables y de esa manera mejorar el estado nutricional.

Todas las actividades desarrolladas por los estudiantes universitarios fueron llevadas adelante de forma conjunta con los docentes y tutores de la cátedra, además de ser consensuadas previamente con las autoridades y docentes de las escuelas con las que se trabajó en esta practica de extensión de educación experiencial. Todo aprendizaje, de cualquier tipo que sea, es para el sujeto una experiencia, algo que le ha ocurrido en su interacción con una situación si es que ha dejado una huella en él. Lo que denominamos educación experiencial se refiere a una clase particular de aprendizaje, a una estrategia de enseñanza con enfoque holístico que está destinada a relacionar el aprendizaje académico con la vida real. Con ese fin, propone al alumno realizar actividades en las que, a partir de esa conexión con la práctica, se le requiere que ponga a prueba en situación auténtica, es decir, de la vida real, las habilidades y los conocimientos teóricos que posee, que evalúe sus consecuencias, enriquezca esos conocimientos y habilidades, identifique nuevos problemas y fije prioridades en cuanto a las urgencias de su solución. Estas formas requerirán: planificación, capacitación, seguimiento del proceso, reflexión sobre las acciones, conocimientos aprendidos y evaluación (Camilloni, 2013).

Desde esta perspectiva, la calidad de la experiencia define la calidad de la enseñanza. Esta concepción la venimos aplicando desde el año 2008 en la asignatura Epidemiología General y Nutricional perteneciente al $3^{\circ}$ año de la carrera de Licenciatura en Nutrición. Desde ese entonces involucramos a los alumnos que cursan año a año la asignatura en una actividad concreta en terreno, a veces en el marco de Proyectos de Extensión que fueron aprobados en convocatorias o como una actividad más de la cátedra. Los resultados de estas experiencias han sido multiplicadores tanto para los alumnos como para los docentes y los destinatarios de las acciones.

En el año 2015, el equipo docente de cátedra presentó y desarrolló por primera vez una propuesta de extensión de educación experiencial. Desde la UNL se concibe a este tipo de prácticas de extensión como "actividades curriculares realizadas por los alumnos que impliquen diversos niveles de interacción e intervención en el medio social, cultural y productivo", ${ }^{1}$ y son enmarcadas en la modalidad de educación experiencial.

Considerando nuevamente a Alicia Camilloni, la extensión puede ser utilizada como componente del currículo, lo cual demanda reflexionar sobre la acción, transformando la experiencia en experiencia de aprendizaje, haciéndola educativa; y como estrategia de enseñanza, un aprender haciendo, con enfoque holístico, destinado a relacionar el aprendizaje con la vida real. En esta dirección, desde el espacio curricular de Epidemiología General y Nutricional, con el fin de que los alumnos puedan relacionar el aprendizaje con una situación en contexto real, se trabajó específicamente con una unidad del programa "Estudios Epidemiológicos de asociación: Corte Transversal”. Este tipo de estudio evalúa situaciones que ocurrieron naturalmente, analiza una población o una muestra de ella en un momento dado, y permite establecer la proporción de población con una determinada condición de salud (estudios de prevalencia). En nuestro caso, la condición de salud analizada por los estudiantes fue la malnutrición de los escolares.

Desde esta práctica los alumnos universitarios pudieron vivenciar cómo es la realización de un estudio transversal directamente en una situación de la vida real en las instituciones escolares y no solamente desde un aspecto teórico, como se desarrollaba en años anteriores desde la asignatura. Participaron desde la planificación, pudieron observar y reflexionar sobre cuáles son los inconvenientes que se van presentando en el desarrollo de la propuesta. Además, a través del proyecto vieron diferentes realidades que se presentan en los distintos sectores de la ciudad, lo que abrió su percepción y los sensibilizó.

La propuesta tuvo como propósito fortalecer acciones interinstitucionales orientadas a contribuir en la incorporación de hábitos saludables y la enseñanza de contenidos relacionados con la nutrición de los niños y niñas de las escuelas seleccionadas de la ciudad de Santa Fe así como a la formación y compromiso social de los estudiantes universitarios.

Los objetivos de aprendizaje que se plantearon y se llevaron adelante de manera conjunta por los estudiantes de nutrición, equipo docente 
y destinatarios, fueron:

- Determinar la condición nutricional de los niños y niñas al ingreso escolar ( $1^{\circ}$ grado) y a mitad de la escuela primaria ( $4^{\circ}$ grado) de los establecimientos educativos seleccionados de la ciudad de Santa Fe. - Vivenciar las problemáticas y desafíos actuales de la enseñanza de la nutrición en escolares de la ciudad de Santa Fe.

- Resolver, en el contexto de las instituciones educativas, situaciones problemas que les promuevan aprendizajes relevantes y puedan realizar un estudio transversal en un contexto real.

- Valorar el trabajo en equipo, a través de actitudes de solidaridad, compromiso y respeto por la tarea de los demás integrantes de la propuesta.

Servicios que se espera del alumno:

- Recolección de los datos antropométricos (peso y talla) en los escolares de $1^{\circ}$ y $4^{\circ}$ grado.

- Determinación de la condición nutricional de los escolares.

- Sistematización de la información recolectada y áreas temáticas de interés con miras a la estructuración del/los temas/s a trabajar de manera conjunta.

- Identificación participativa de los intereses y expectativas de los niños y niñas.

- Diseño colectivo de propuesta/s que para el mejoramiento de la condición nutricional de los niños y niñas con trastornos nutricionales de las escuelas seleccionadas.

Algunos de los servicios esperados que el alumno desarrolle son una parte fundamental para la construcción de un Sistema de vigilancia alimentaria y nutricional, ya que los alumnos realizan la recolección, análisis, interpretación y difusión de datos, pero lo hacen para un año en particular como parte de una actividad del programa de la asignatura, que es la aplicación de un estudio de corte transversal. El proceso se vuelve sistemático a través de los alumnos que cursan año tras año la asignatura; es el equipo docente el que finalmente lleva adelante la vigilancia nutricional y esto permite luego poder observar las tendencias en tiempo, lugar y persona, con lo que se observan y anticipan cambios para realizar las acciones oportunas, incluyendo la investigación y/o la aplicación de las medidas de control de los problemas alimentarios y nutricionales. Esto sería un claro ejemplo de cómo desde la cátedra de Epidemiología y Salud Pública se trabaja en la integración de la docencia, la extensión y la investigación.

De esta experiencia realizada durante 2015 participaron 54 alumnos de la Licenciatura en Nutrición que cursaron la asignatura Epidemiología General y Nutricional, el equipo de docentes extensionistas, 1 graduada y 1 alumna avanzada de la carrera que actuaron como tutores, docentes y directivos de las escuelas, 771 niños y niñas de instituciones educativas ${ }^{2}$ de la ciudad de Santa Fe. Estas escuelas fueron seleccionadas por un muestreo aleatorio del total de instituciones escolares con las que se viene trabajando de manera conjunta desde la cátedra desde el año 2010.

Además se trabajó de manera indirecta con los padres de estos niños y niñas.

\section{Conexión de la teoría con la realidad}

Consideramos que participar en actividades de extensión es el impulso inicial para movilizar procesos de formación auténtica (Brown, 1993). Entendemos la extensión universitaria como espacio de prácticas sociales compartidas y colaborativas para la construcción de propuestas alternativas. Estas actividades se consolidan mediante las acciones conjuntas que se tejen en los escenarios sociales/culturales/históricos con el propósito de comprender e intervenir de manera creativa e innovadora en las situaciones identificadas como problemas sociales (Rafaghelli, 2013). En este sentido, desde el equipo docente se trató de trabajar y hacer partícipes a los alumnos de todas las etapas del proyecto: preliminar, implementación en terreno y posterior, para que ellos pudieran tener en claro cuáles eran los contenidos teóricos que estaban aplicando en su práctica de educación experiencial. Se decidió modificar el Cronograma Académico de clases adelantando algunos temas, por ejemplo: Estudios de prevalencia y estudios transversales, para que ellos puedan ver la aplicación de realizar un estudio epidemiológico transversal en las escuelas donde se estaba realizando la práctica. Asimismo, en el transcurso de todas las clases teóricas se utilizaban como ejemplos de aplicación o adaptando los problemas a la situación en contexto que se estaba desarrollando con los niños de las escuelas primarias de la ciudad de Santa Fe. En esta dirección, se desarrollaron actividades intra y extraáulicas que prepararon a los estudiantes en las capacidades y conocimientos técnicos específicos, así como en las capacidades actitudinales requeridas para el diálogo con la comunidad educativa.

\section{Evaluación de la experiencia}

Durante la última etapa de desarrollo de la propuesta, los alumnos realizaron un informe final individual donde pudieron expresar su evaluación sobre el trabajo realizado en el marco de la educación experiencial en la asignatura Epidemiología General y Nutricional. La mayoría de los alumnos de Licenciatura en Nutrición evaluó su desempeño en relación con el trabajo técnico-académico como $\mathrm{n}^{\circ} 6 \mathrm{Dr}$. Mariano Moreno. Escuela $\mathrm{n}^{\circ} 5$ Vicente López y Planes. Escuela $n^{\circ} 14$
Dr. Nicolás Avellaneda. Escuela $\mathrm{n}^{\circ} 1102$ Don Bosco. Colegio La Salle Jobson.
Escuela $n^{\circ} 18$ Falucho. Escuela $n^{\circ} 869$ Julio Argentino Roca. 
bueno; en mayor proporción destacan la capacidad de organización del trabajo y la de gestión de la información. En la Tabla $n^{\circ} 1$ se observa que "la posibilidad de aplicación de conocimientos previos" es la actividad que se diferencia de las demás, debido a que es mayor la proporción de alumnos que la evaluó como muy buena. Por otro lado, la mayoría de los alumnos evalúo su desempeño en cuanto al desarrollo personal (compromiso y actitud) entre muy bueno y bueno; en mayor proporción destacan el compromiso ético como muy bueno y la capacidad para reconsiderar las posiciones propias en el diálogo durante la realización de actividades particulares previstas como buena (Gráfico $n^{\circ} 1$ ).

Respecto del trabajo colaborativo, para la mayoría de los alumnos era su primera participación en una práctica de extensión, sólo 2 del total de los alumnos habían participado en un proyecto de extensión en una oportunidad anterior. Por eso lo pudieron hacer, siempre esperando las indicaciones y aclaraciones de los docentes y/o tutores.

De las respuestas de las evaluaciones de los informes de los alumnos, en cuanto a la capacidad de organización del trabajo, un $32 \%$ la evaluó como muy buena, un 66 \% como buena, y sólo un $2 \%$ como regular. Respecto de la capacidad de ejecución de las acciones planificadas, $40 \%$ de los alumnos la evalúo como muy buena, 57 \% como buena, y el $3 \%$ regular. También fueron consultados sobre la capacidad para interactuar con grupos heterogéneos, y las respuestas estuvieron repartidas equitativamente entre buena y muy buena.

Respecto de la adaptación al territorio, desde el equipo docente la evaluamos como muy buena y a la vez enriquecedora. Todos los alumnos pudieron participar de esta experiencia de aprendizaje servicio, y eso permitió que pusieran en juego sus conocimientos académicos para el abordaje de un problema en contextos reales al servicio de otros actores sociales de la comunidad, que para nuestro caso serían los niños/as, los padres, los docentes y directivos de las escuelas en las cuales se desarrolló la práctica de extensión.

De las respuestas de las evaluaciones de los informes de los alumnos, en cuanto a la posibilidad de adaptación a nuevas situaciones, el $43 \%$ de los alumnos la evaluó como muy buena, y un $57 \%$ como buena. A la adaptación al terreno la evaluaron como muy buena en un $47 \%$, y como buena un $53 \%$ de ellos. Además, se pidió a los alumnos que evaluaran su compromiso ético, el $72 \%$ de ellos lo calificó como muy bueno y el $28 \%$ restante como bueno.

\subsection{Dificultades encontradas}

Las dificultades encontradas estuvieron relacionadas, por un lado, con la posibilidad de adaptarse a la situación en contexto y, por el otro, con la falta de coordinación entre las autoridades de la escuela y los docentes a cargo de los niños.

Para poder realizar las mediciones antropométricas por parte de nuestros estudiantes se necesita coordinar que ocurran varias cosas en simultáneo, entre ellas:

1. Tener el consentimiento informado firmado por el padre y/o tutor del niño.

2. Que el niño se lo haya entregado a la docente.

3. Que la docente haya entregado todos los consentimientos a la directora de la escuela.

4. Que el niño/a asista (tenga el presente) a la escuela el día que se coordinó previamente con la autoridad de la misma.

5. Que el día de la implementación de la práctica en terreno los niños no tengan un paseo o una actividad fuera de la escuela. 6. Que el lugar que se nos asigna en la escuela tenga el espacio suficiente para realizar las mediciones y que se hagan de a un niño/a por vez.

Gráfico $n^{\circ}$ 1. Evaluación del desempeño en relación al desarrollo personal. Alumnos $3^{\circ}$ año Licenciatura en Nutrición FBCB-UNL

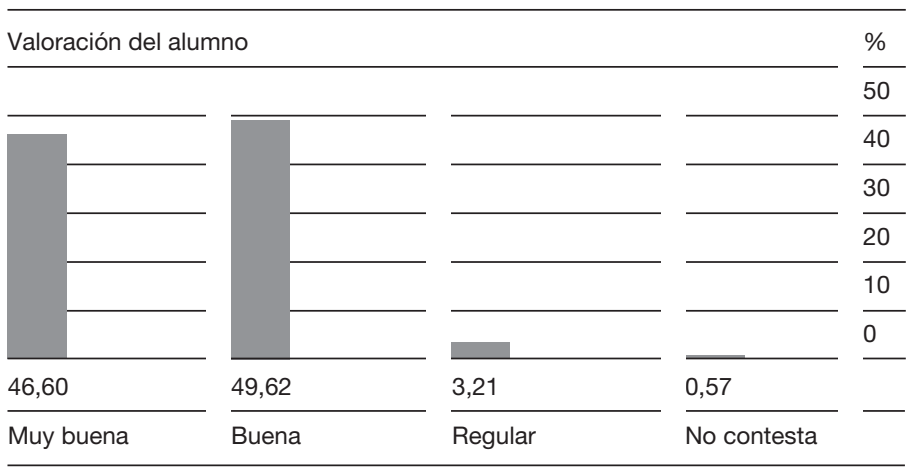

Tabla $n^{\circ}$ 1. Evaluación del desempeño en relación con el trabajo técnico-académico que realizó. Alumnos $3^{\circ}$ año Licenciatura en Nutrición FBCB-UNL

\begin{tabular}{|c|c|c|c|c|}
\hline Actividad & $\begin{array}{l}\text { Muy Bueno (MB) } \\
(\%)\end{array}$ & $\begin{array}{l}\text { Bueno (B) } \\
(\%)\end{array}$ & $\begin{array}{l}\text { Regular (R) } \\
(\%)\end{array}$ & $\begin{array}{l}\text { Malo (M) } \\
(\%)\end{array}$ \\
\hline Posibilidad de aplicación de conocimientos previos & 49,06 & 47,17 & 3,77 & - \\
\hline Posibilidad de adaptación a nuevas situaciones & 43,40 & 56,60 & - & - \\
\hline Capacidad de organización del trabajo & 32,08 & 66,04 & 1,89 & - \\
\hline Capacidad de gestión de información & 37,74 & 60,38 & 1,89 & - \\
\hline Capacidad de ejecución de las acciones planificadas & 39,62 & 56,6 & 3,77 & - \\
\hline
\end{tabular}


Éstos son solamente algunos ejemplos de todo lo que sería necesario para que la experiencia se pueda desarrollar correctamente o en las condiciones teóricas esperadas de un estudio transversal.

En el informe de los alumnos también se les consultó sobre las dificultades encontradas, y podemos citar con frases significativas, según sus propias palabras, lo que ellos mencionan al respecto:

- "Las autoridades de la escuela no enviaron los consentimientos informados con anterioridad, por lo cual algunos debieron ir al establecimiento reiteradas veces".

- "Muy pocos padres habían firmado el consentimiento informado, por lo cual se pudieron evaluar pocos alumnos."

- "Las autoridades de las escuelas o los docentes se negaban a entregar los informes a los padres porque su opinión no coincidía con los resultados obtenidos por nosotros."

- "Las docentes de la escuela estaban muy apuradas para que se termine con la actividad por lo que enviaban muchos chicos en corto período de tiempo (...) dejaban el curso entero en la sala en la cual estábamos desempeñando nuestra actividad, los niños estaban muy inquietos y no podíamos realizar un correcto desempeño de nuestra labor debido a que no podíamos tener una correcta comunicación."

- "Los compañeros que realizaban las medidas antropométricas lo hacían demasiado rápido y los que íbamos anotando no llegábamos a escribir."

A medida que iban surgiendo inconvenientes se buscaba una manera de solucionarlos para que no volvieran a ocurrir en el siguiente encuentro en el establecimiento escolar. Por ejemplo, con anterioridad a la asistencia a la escuela se contactaba telefónicamente para recordar a autoridades y docentes la necesidad e importancia de contar con los consentimientos informados de los padres para hacer la valoración nutricional. En el momento de la actividad en terreno, se conversaba con las docentes para sacar del aula a los niños y niñas en pequeños grupos y realizar la misma de manera más organizada.

Todas las dificultades pudieron ser solucionadas, pero quedaron niños que no fueron medidos ya que no asistieron el día que se realizó la práctica en la escuela.

\subsection{Valores que estuvieron presentes en el desarrollo del proyecto}

La experiencia de educación experiencial propicia aprendizajes profundos que solo se construyen en la realización de actividades en los contextos reales de prácticas sociales. Consideramos que los alumnos trabajaron durante todas las instancias del proyecto con responsabilidad ética y los valores que estuvieron presentes fueron: compromiso, responsabilidad, respeto, solidaridad y compañerismo.

\subsection{Aspectos más significativos de esta experiencia} Para el equipo docente fue una experiencia muy significativa ya que es la primera vez que se planteó el dictado de la asignatura con la experiencia de aprendizaje servicio y se involucró a todos los alumnos en todas las instancias del proyecto. Significó poder adaptar el programa y el cronograma de la asignatura a esta práctica de educación experiencial, para lo cual también se sostuvieron a lo largo de todo el cuatrimestre (e incluso con anterioridad al mismo) reuniones de cátedra todas las semanas. Desde el equipo se sostiene que esta manera de aprender en servicio es un compromiso social de quienes estamos en la Universidad y, por lo tanto, es necesario propiciar este tipo de instancias para que los estudiantes lo comprendan y puedan participar. Estas instancias no pueden quedar por fuera del curriculum sino que deben ser parte constitutiva del mismo. En el informe final de los alumnos, al ser consultados sobre los aspectos más significativos, también expresaron conceptos como: - "Observación directa de la realidad".

- "La aplicación de conocimientos teóricos a situaciones reales fuera de la Facultad y el trabajo realizado en equipo."

- "La primera experiencia en el terreno."

- "Interiorizarse con lo que va a ser el trabajo del licenciado en Nutrición."

- "El contacto con los niños, la buena predisposición de ellos para ser evaluados y el interés demostrado por la actividad."

- "Evaluar la condición nutricional en escolares (medición de peso y talla)."

- "La diferencia de resultados obtenidos entre las escuelas analizadas."

- "Transmitirles e informar tanto a los escolares, como a los padres, la importancia de una buena alimentación y hábitos saludables."

Desde el ámbito escolar se pudo conocer la situación real del estado nutricional de los niños. Como se mencionó, en los resultados obtenidos existe una alta prevalencia de malnutrición por exceso (sobrepeso y obesidad) en las niñas y niños de las escuelas con las que se trabajó en esta experiencia.

Dado que tanto el sobrepeso como la obesidad se asocian a una mayor ocurrencia de problemas de salud en la juventud y en la edad adulta, todos los países están poniendo en práctica acciones para prevenir el aumento de peso en las edades más jóvenes y en la edad escolar.

Se hace necesario poder continuar con diferentes acciones tendientes a contribuir de manera conjunta y a mejorar la condición nutricional de los escolares. 


\section{A modo de conclusión}

Desde el equipo docente creemos que las prácticas de educación experiencial deberían estar siempre presentes en el aprendizaje y en la enseñanza de todos los alumnos y docentes de Epidemiología General y Nutricional. Sería deseable que los alumnos de la carrera tuvieran este tipo de prácticas durante todo el cursado para llegar a ser mejores profesionales en un futuro cercano.

Hay contenidos que un alumno aprende y que el docente enseña en este tipo de experiencias que va más allá del contenido analítico del programa de la asignatura y que tiene que ver con la formación como personas comprometidas con el contexto social en el que viven.

En el informe final del alumno también se les consultó sobre algún comentario o sugerencia adicional, y aquí presentamos algunas de sus expresiones:

- "Fue una hermosa experiencia."

- "No hay muchas materias que realizan ese tipo de actividades, por lo cual es muy importante que se siga llevando a cabo para el progreso de los próximos estudiantes."

- "La actividad llevada a cabo fue muy enriquecedora."

- "Fue muy bueno el trabajo en conjunto con los ayudantes de la cátedra."

- "La dedicación que ponen los profesores en el desarrollo de la materia (puntualidad, material, información, prolijidad)."
A partir de esta propuesta de educación experiencial, dado que se obtuvo una alta prevalencia de malnutrición por exceso en niños y niñas que asisten a escuelas de la ciudad de Santa Fe con las que se trabajó en este proyecto, se presentaron los resultados a las autoridades de la Sociedad Argentina de Pediatría filial Santa Fe y a la Dirección de Maternidad e Infancia del Ministerio de Salud de la Nación.

Para abordar la problemática de la malnutrición por exceso se está promoviendo que las personas, las familias y la comunidad adopten o refuercen hábitos saludables de alimentación y de actividad física, ya que esto ha demostrado ser la estrategia más eficaz en Salud Pública para mejorar la condición nutricional de la población. Pero como el cambio de conductas, tanto en lo que refiere a los hábitos de alimentación (para hacer que sean más saludables) como a la actividad física, lleva tiempo, la detección más temprana posible de una condición nutricional de exceso de peso permite poner en práctica rápidamente estrategias que promuevan este cambio de hábitos antes de que sea más difícil revertir la situación. Por ello es que la Organización Mundial de la Salud, la Sociedad Argentina de Pediatría y el Ministerio de Salud de la Nación proponen detectar pequeñas desviaciones del estado nutricional normal en la niñez y adolescencia, para brindar al personal de salud y a las familias mayor tiempo y posibilidades de mejorar el estado nutricional del niño.

\title{
66
}

\author{
desde el ámbito escolar se pudo \\ conocer la situación real del \\ estado nutricional de los niños
}

\section{Referencias bibliográficas}

Brown y otros (1993). Conocimiento especializado distribuido en el aula. En Salomon, G. (Comp.), Cogniciones distribuidas. Consideraciones psicológicas y educativas. Buenos Aires: Amorrortu.

Camilloni, A. (2013). La inclusión de la educación experiencial en el currículo universitario. En: Menéndez, G. y otros. Integración docencia y extensión. Otra forma de enseñar y de aprender. Santa Fe: Ediciones UNL.

Last John, M. (2001). Dictionary of epidemiology. Fourth edition. Oxford: American Journal Epidemiology.

Ministerio de Salud de la Nación. Dirección de Epidemiología (2013). Guía para el fortalecimiento de la Vigilancia de la Salud en el nivel local. Buenos Aires.

Rafaghelli, M. (2013). La dimensión pedagógica de la extensión. En Menéndez, G. y otros. Integración docencia y extensión. Otra forma de enseñar y de aprender.

Santa Fe: Ediciones UNL.

Universidad Nacional del Litoral (2007). Resolución Honorable Consejo Superior $n^{\circ}$ 274. 2007. Santa Fe, Argentina. 